\title{
Determination of Mesalazine Using Terbium Rare Earth lons as a Fluorescence Probe
}

\author{
Yu-Guang LV ${ }^{1}$, a*, Di SONG ${ }^{1}$, Du A ${ }^{1}$, Ji-Hui ZHENG ${ }^{1}$, Hong-Bing GAO ${ }^{1}$,
} Xiao-Li Q1 ${ }^{2, b}$, Jiang $\mathrm{WU}^{3}$, Zhong-Ping GONG ${ }^{1}$, Jie TENG

${ }^{1}$ College of Pharmacy, Jiamusi University, Jiamusi 154007, China

${ }^{2}$ College of Life Sciences, Jiamusi University, Jiamusi 154007, China

${ }^{3}$ School of Stomatology, Jiamusi University, Heilongjiang 154002, China

${ }^{4}$ Tianjin Key Laboratory on Technologies Enabling Development of Clinical Therapeutics and Diagnostics (Theranostics), School of Pharmacy, Tianjin Medical University, Tianjin 300070, China.

a yuguanglv@163.com, bqixiaoli3@tom.com

${ }^{*}$ Corresponding author

Keywords: Ulcerative Colitis, Mesalazine, Terbium Rare Earth, Fluorescent, Content Determination.

\begin{abstract}
Ulcerative colitis is chronic nonspecific colitis. Now,the main first-line drugs are preparations with mesalazine. Chemical name of mesalazine is 5- aminosalicylic acid (5-ASA), which one of colon target drugs has function of anti-inflammatory by inhibit process of leukotriene, prostaglandin $\mathrm{E}(\mathrm{PG})$ and free radical. Comparing with traditional SASP drugs used for treating colitis, 5-ASA has character of less harmful to body. In recent decade, mechanism and preparation of the mesalazine have attended at home and abroad. This paper established the rare earth fluorescence probe, which a new kind of technology for drug determination, contenting 5-ASA. It is more convenient, shortcut, accuracy than common methods of titration, ultraviolet spectrophotometry and high-performance liquid chromatography. According this, it provided a new and viable method of determination of 5-ASA. This method also can assist to research 5-ASA in the subjects that biopharmaceutical analysis, pharmacokinetics and clinical pharmacy, required strict experiment conditions.
\end{abstract}

\section{Introduction}

Ulcerative colitis is a chronic nonspecific disease of colon, main lesion sit was colonic and rectal mucosa. Its mechanism and pathogeny are complex. Now, there is a generally believed that it is caused by appropriate autoimmune[1]. The first-line drugs for treating ulcerative colitis are preparations composed of 5-amino salicylic acid (5-ASA).5-ASA is also called mesalamine, white or light gray white needle-shaped crystal, m.p.is $270 \sim 275^{\circ} \mathrm{C}$, easy to oxidation, color may darken on exposure to air and slightly soluble in water. Solubility in water $\left(37^{\circ} \mathrm{C}\right)$ is $1.41 \mathrm{~g} / \mathrm{L}$ and its aqueous solution is partial acid. The $\mathrm{pH}$ of the saturated solution $\left(25^{\circ} \mathrm{C}\right)$ was 4.1 . Very slightly soluble in ethanol, acetone and methanol.Hardly insoluble in chloroform, ethyl acetate and n-hexane. When $\mathrm{pH}<2.0$ or $\mathrm{pH}>5.5$, the solubility of 5-ASA significantly increased. Anti-inflammatory work by inhibiting to produce prostaglandin E, leukotrienes, and free radicals. Its role is partial, in other word, it works when touched or complexed with the mucosa and without effect depend on blood circulation. Oral administration can be quickly absorbed in stomach and small intestine, not having enough drugs to the colon for effecting of anti-inflammatory[2]. Therefore, oral 5-ASA mainly made two kinds of preparations for improving drug utilization and reducing body injurious. The one is prodrugs, which are controlled-release under the action of special enzymes and not absorbed in stomach and small intestine such as Balyua(basalazine)by Astra company which was listed in its first market UK at 1997, domestic production Balsalazide Sodium Tablets(Bei Si Le), Olsalazine sodium capsules (Chang Mei, Pastan) by Austrian. The second is coated drugs. Which can release in special position,e,g. Salofak by Germany Hawke, Pentasa by Germany Ferring, Etiasa by France 
Ipsen, Asaco by British Tillotts[3], domestic production Hui Di. In addition, Kim H[4], et al. are being studied and synthesis of 5 - amino salicylic acid glycine salts, which have great potential in position colon. Jung[5], et al. study 5 - amino salicylic acid glutamate and aspartate, found out that two derivatives do not decompose in the stomach and small intestine, can be passed to the colon.

Wang Guijie, LiYan[6], from Shengjing Hospital of China Medical University, through clinical research found out mesalazine have less untoward effects than SASP (one of sulfonamide clinical drugs for commonly treating ulcerative colitis), in the treatment of ulcerative colitis. In addition, there is no significant difference side effects on the rash, but others such as leukopenia, liver function abnormalities, nausea, vomiting and gastrointestinal side effects are obvious different. This result is similar as domestic and foreign have reported[7, 8].Some foreign experts also found that mesalazine in the treatment of colitis for pregnant women and infants, have less harmful and can be used in drug combination. The effect is obvious and worthy of promotion [9-14]. In this paper, studying on choosing 5-ASA complex with $\mathrm{Tb}^{3+}$ rare earth ions. Optimizing experimental conditions, applying this method to detect coated and un coated tablets, and comparing with the current national standard (Ch.p.2010) [15-17].This method is simple, accurate, feasible and high sensitivity, can be successful applied to the determination of mesalazine.

\section{Experimental Method}

To $10 \mathrm{~mL}$ color comparison tubes,solutions were added in the following order: $\mathrm{Tb}^{3+}$ solution, 5-ASA solution, Tris- $\mathrm{HCl}\left(0.1 \mathrm{~mol}^{-1} \mathrm{~L}^{-1} \mathrm{pH}=9.0\right)$ buffer solution, SDBS solution. The mixture was volumed to the mark with redistilled water.Shaking and standing at room temperature for $10 \mathrm{~min}$, the fluorescence intensity were measured at $\lambda_{\mathrm{ex}} / \lambda_{\mathrm{em}}=373 \mathrm{~nm} / 545 \mathrm{~nm}, 1 \mathrm{~cm}$ fluorescence cuvette, slit width of excitation and emission both are $10 \mathrm{~nm}$.

\section{Results and Discussion}

Pure $\mathrm{Tb}^{3+}$ solution had not shown any characteristic fluorescence spectrum, but $5-\mathrm{ASA}_{-} \mathrm{Tb}^{3+}$ complex solution showed characteristic fluorescence spectrum of $\mathrm{Tb}^{3+}$. 5-ASA itself characteristic intensity significantly reduced at $435 \mathrm{~nm}$ (Fig.1).

Analysis this phenomenon,this may indicate that the molecule of 5-ASA absorbed ultraviolet for $\pi \sim \pi^{*}$ translation. Exciting molecule conveyed the energy to $\mathrm{Tb}^{3+}$ by coupling resonance with ${ }^{5} \mathrm{D}_{4}$ energy level of coordinated $\mathrm{Tb}^{3+}$, which is a form of non-radiative, in the molecule internal. Then $\mathrm{Tb}^{3+}$ emitted fluorescence by ${ }^{5} \mathrm{D}_{4} \rightarrow{ }^{7} \mathrm{~F}_{\mathrm{J}}$ translation. i.e., Antenna effect. Actually, only little fluorescence energy from $\mathrm{Tb}^{3+}$ self absorbed ultraviolet, most part from coordinations
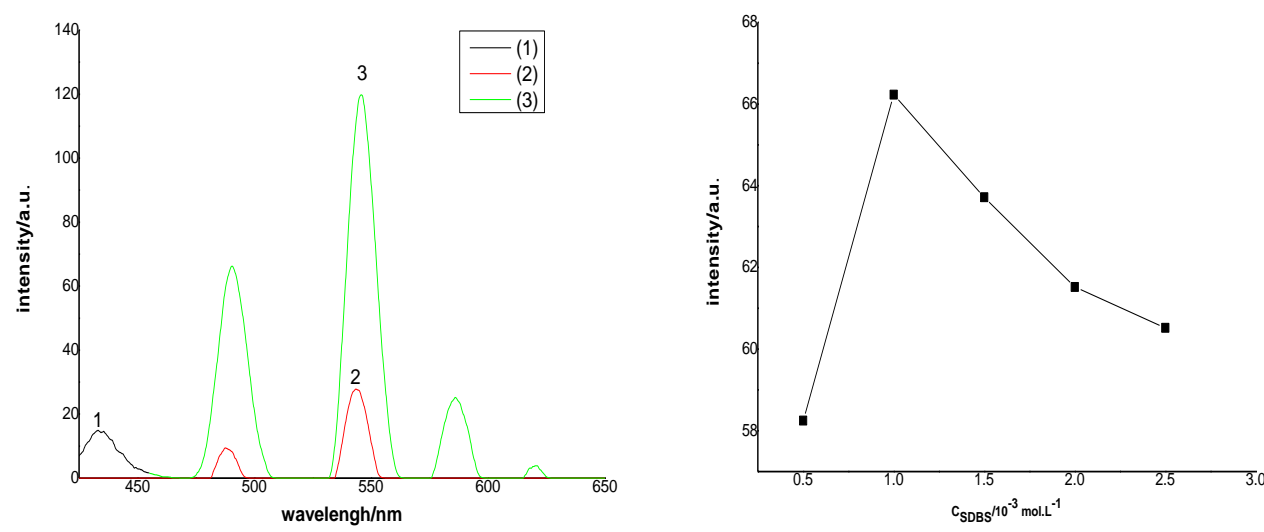

1.5-ASA; $2 . \mathrm{Tb}^{3+}+5-\mathrm{ASA} ; 3 . \mathrm{Tb}^{3+}+5-\mathrm{ASA}+\mathrm{SDBS} ; \quad \mathrm{C}_{\mathrm{Tb} 3+}=3 \times 10^{-3} \mathrm{~mol} \cdot \mathrm{L}^{-1}, \mathrm{C}_{5-\mathrm{ASA}}=1 \times 10^{-5} \mathrm{~mol} . \mathrm{L}^{-1}, \mathrm{pH}=6.5$ $\mathrm{C}_{\mathrm{Tb} 3+}=3 \times 10^{-3} \mathrm{~mol} . \mathrm{L}^{-1}, \mathrm{C}_{5-\mathrm{ASA}}=1 \times 10^{-5} \mathrm{~mol} . \mathrm{L}^{-1}$,

$\mathrm{C}_{\mathrm{SDBS}}=1 \times 10^{-3} \mathrm{~mol} \cdot \mathrm{L}^{-1}, \mathrm{pH}=6.5$

Fig. 1 The fluorescence emission spectrum curve Fig. 2 Effect of the concentration of the surfactant of 5-ASA rare earth probe on the fluorescence intensity 


\section{Effect of Surfactant}

Although 5-ASA- $\mathrm{Tb}^{3+}$ complex solution showed characteristicof $\mathrm{Tb}^{3+}$ fluorescence, but the signal is weak, adding an appropriate amount of surfactant concentrations greatly enhanced the signal and can be used for quantitative detection(Fig.1).

Surfactant is a molecule composed of two exactly different particles,one is lipophilic and the other is hydrophilic. After it dissolve in water, can reduce the surface tension of water and improve solubility of the organic compounds.

This experiment investigated the effect of different surfactants on the fluorescence intensity, and the result was found to be positive, the effects of different types of surfactants, including non-ionic surfactants solid (GA, $\beta$-CD), a cationic surfactant (CTMAB) and anionic surfactants (SDS, SDBS). GA, $\beta$-CD and CTMAB sensitizing strength was limited and image effect was not good, while the anionic surfactants SDS and SDBS are better, the SDBS is the most effective surfactant, so in this study, SDBS was used as a sensitizing surfactant.

SDBS hydrophilic group is a sulfonic acid group which enveloped fluorescence molecule in the system and prevented action with water molecule. Its lipophilic group is alkyl group which adjoin fluorescence molecule. SDBS would form micelles like a package contains fluorescence compounds. This space existence can reduce quenching by O-H vibration of water molecule. On the other hand, fluorescence molecule gathered, according to Traube's Rule(when solution at a low concentration, its surface tension decline proportion to its concentration), would lead to surface tension of the system decline and fluorescence intensity enhance.

\section{Effect of the Concentration of the Surfactant}

As shown in Fig.2, SDBS concentration has a significant effect on the fluorescence intensity. The fluorescence intensity was largest when the concentration of SDBS was $1.0 \times 10^{-3} \mathrm{~mol}^{-\mathrm{L}^{-1}}$. In this experiment, $1 \times 10^{-3} \mathrm{~mol} . \mathrm{L}^{-1}$ was chosen for the further study.

\section{PH Effect}

5-ASA solubility is minimum in the $\mathrm{pH}$ range 2.0 to 5.5[2]. In the environment of strong acid, fluorescence intensity be effect by $\mathrm{H}^{+}$and hydrogen bonds. In the environment of strong alkali, $\mathrm{Tb}^{3+}$ occurs hydrolysis reaction produce $\mathrm{Tb}(\mathrm{OH})_{3}$. Therefore, this paper study on 5-ASA-Tb ${ }^{3+}$-SDBS fluorescence intensity of the system at $\mathrm{pH}$ 5.0-9.0. The results as Fig. 3. In Fig. 3, it is showed that the effect of different $\mathrm{pH}$ on 5-ASA-Tb ${ }^{3+}$-SDBS fluorescence intensity at 5.5 9.0. As shown, $\mathrm{pH}$ between 5.0 and 6.5 continued to enhance the fluorescence intensity, the largest intensity was observed at $\mathrm{pH}=6.5$. So $\mathrm{pH}=6.5$ was used in the following experiment.
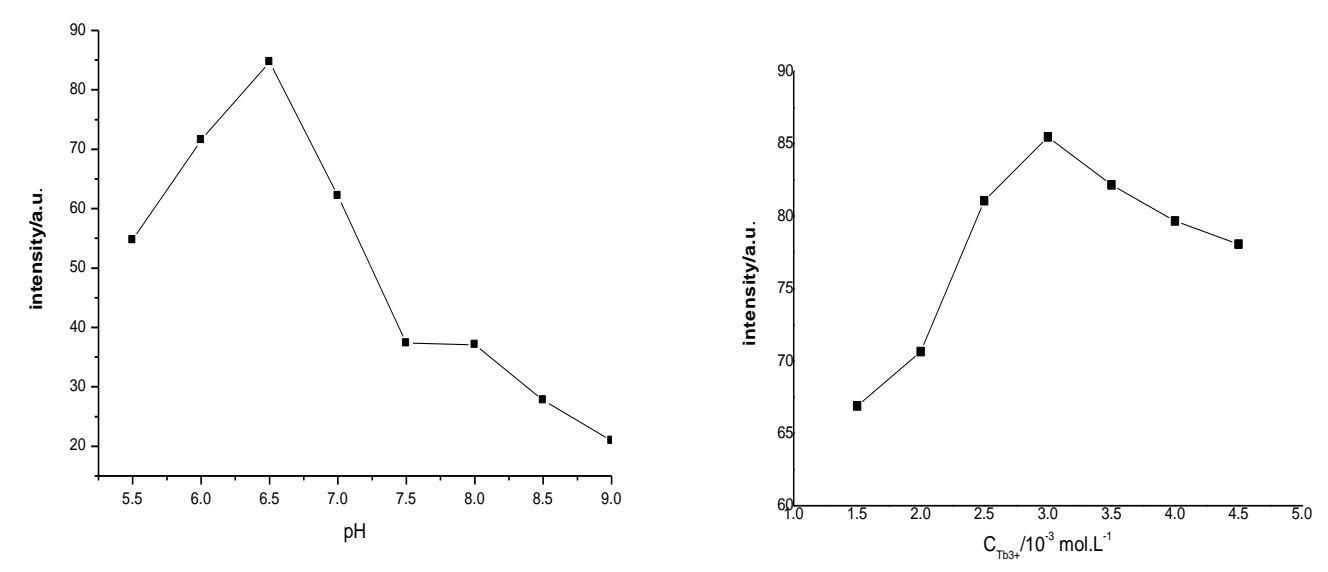

$\mathrm{C}_{\mathrm{Tb} 3+}=3 \times 10^{-3} \mathrm{~mol} \cdot \mathrm{L}^{-1}, \mathrm{C}_{5-\mathrm{ASA}}=1 \times 10^{-5} \mathrm{~mol} \cdot \mathrm{L}^{-1}, \mathrm{C}_{\mathrm{SDBS}}=1 \times 10^{-3} \mathrm{~mol} \cdot \mathrm{L}^{-1}$

$\mathrm{C}_{5-\mathrm{ASA}}=1 \times 10^{-5} \mathrm{~mol} . \mathrm{L}^{-1}, \mathrm{C}_{\mathrm{SDBS}}=1 \times 10^{-3} \mathrm{~mol} . \mathrm{L}^{-1}, \mathrm{pH}=6.5$

Fig. 3 Effect of $\mathrm{pH}$ on the Fluorescence Intensity Fig.4 Effect of Rare Earth $\mathrm{Tb}^{3+}$ Concentration on Fluorescence Intensity 
We also found out that by using kinds of different buffers to set $\mathrm{pH}=6.5$, fluorescence intensity of system is not same. The follow buffers are uesd in the experiment: Tris- $\mathrm{HCl}, \mathrm{NH}_{4} \mathrm{Ac}-\mathrm{HAc}$, $\mathrm{NaAc}-\mathrm{HA}$ and $\mathrm{NH}_{4} \mathrm{Cl}-\mathrm{HCl}$. The results of experiment showed that 0.1 mol.L $\mathrm{L}^{-1}$ Tris- $\mathrm{HCl}$ buffer solution has the largest intensity and $0.1 \mathrm{~mol}^{-\mathrm{L}^{-1}}$ Tris- $\mathrm{HCl}$ buffer solution should be chosen in this experiment.

\section{Effect of $\mathbf{T b}^{3+}$ Concentration.}

In the condition of fixed 5-ASA concentration, SDBS concentration used was $1.0 \times 10-3 \mathrm{~mol}$.L- 1 and Tris- $\mathrm{HCl}$ buffer solution concentration used was $0.1 \mathrm{~mol} . \mathrm{L}^{-1}, \mathrm{pH}=6.5$, study on fluorescence intensity related to different concentrations of $\mathrm{Tb}^{3+}$ solution .

Studies have found that when the concentrations of $\mathrm{Tb}^{3+}$ is between $2.5 \times 10^{-3} \mathrm{~mol}$. $\mathrm{L}^{-1} \sim 3.5 \times 10^{-3} \mathrm{~mol} . \mathrm{L}^{-1}$, would shown strong fluorescence intensity. Therefore, $3 \times 10^{-3} \mathrm{~mol} . \mathrm{L}^{-1}$ was used in this experiment(Fig. 4).

\section{Accuracy and Stability Test}

Under the best conditions above test:0.1mol.L-1Tris-HCl, $\mathrm{pH}=6.5,3.0 \times 10^{-2} \mathrm{~mol} . \mathrm{L}^{-1} \mathrm{~Tb}^{3+}$ solution, $1.0 \times 10^{-3} \mathrm{~mol} . \mathrm{L}^{-1} \mathrm{SDBS}$ solution. And parallel detect $1 \times 10^{-5} \mathrm{~mol} . \mathrm{L}^{-1} 5$ - ASA ten times, $\mathrm{RSD} \%=0.867$.

Study on reaction time related to stability of system, it is shown that the system have maximum fluorescence intensity and stability range from $5 \mathrm{~min}$ to $10 \mathrm{~min}$.In this experiment, we chose to stand in room temperature for $5 \mathrm{~min}$ before experiment and finish it within $10 \mathrm{~min}$.

\section{Linear Range and Detection Limits}

Under optimal experimental conditions, the fluorescence intensity have a good liner relationship with 5-ASA which concentration in the range of $6 \times 10^{-7} \sim 3 \times 10^{-5}$ mol. $\mathrm{L}^{-1}$. Linear equation: If $=$ $1.9328 \mathrm{C}+101.7488, \mathrm{r}=0.9999$.

\section{Sample Analysis}

The developed was applied to the determination of 5-ASA tablets. Choosing at randomly, five groups of different batch number of Hui Di tablets by Sunflower pharmaceutical Co.,Ltd.(labeled amount of 5-ASA is $0.25 \mathrm{~g}$ per tablet). Simultaneously, follow the same way, choosing five groups of Hui Di tablets before coated.

Uncoated tablet finely triturated and weighed before dissolved in dilute hydrochloric acid solution, then dilute with redistilled water volume $100 \mathrm{~mL}$ volumetric flask and ultrasonic assist dissolution. After nature sedimentation, $1 \mathrm{~mL}$ was taken from the supernatant to volume another $100 \mathrm{~mL}$ flask. According to the standard curve of this experiment content 5-ASA and do recovery experiments. Coated tablet immersed in pure alcohol for cleaning coating substance on the surface and stoved it. The next steps as same as the uncoated tablet processed method.

According to Ch.P.(2010), using titration method to content coasted tablet and uncoated tablet as controlled experiment. The results are shown in Table 1 , Table $2 . \mathrm{RSD}_{1} \%=0.485(\mathrm{n}=5), \mathrm{RSD}_{2} \%$ $=0.283(\mathrm{n}=5)$.

Tab. 1 Determination of the Raw 5-ASA Tablets

\begin{tabular}{|cccc|}
\hline Samples & Method $(\mathrm{g} / \mathrm{p})$ & Pharmacopoeia $(\mathrm{g} / \mathrm{p})$ & RSD $(\%)$ \\
\hline 1 & 0.255 & 0.254 & 100.4 \\
2 & 0.253 & 0.254 & 99.6 \\
3 & 0.253 & 0.253 & 100.0 \\
4 & 0.252 & 0.250 & 100.8 \\
5 & 0.252 & 0.253 & 99.6 \\
\hline
\end{tabular}


Tab. 2 Determination of 5-ASA Coated Tablets

\begin{tabular}{|c|c|c|c|}
\hline Samples & Method $(\mathrm{g} / \mathrm{p})$ & Pharmacopoeia $(\mathrm{g} / \mathrm{p})$ & $\operatorname{RSD}(\%)$ \\
\hline 1 & 0.251 & 0.251 & 100.0 \\
\hline 2 & 0.251 & 0.250 & 100.4 \\
\hline 3 & 0.251 & 0.250 & 100.4 \\
\hline 4 & 0.252 & 0.251 & 100.4 \\
\hline 5 & 0.250 & 0.251 & 99.6 \\
\hline
\end{tabular}

\section{Conclusions}

This paper established a new method of rare earth fluorescence probe for determination of 5-ASA. Compared with the traditional methods, e.g., titration, HPLC, UV, It have characters of convenient and quick.5-ASA preparations mostly are targeted intestine, with obvious effects and small body injuries, its positioning mechanism and vivo metabolism attracted widespread attention in the subjects of vivo pharmaceutical analysis, pharmacokinetics and pharmaceutical at home and abroad. But investigations in pharmaceutical analysis were reported not much.

This new method is environmental friendly. Less demanding solvent and less using reagents. Therefore, the future would be brilliant if this method be used in 5-ASA targeted research. But it still need medicine workers make joint efforts.

\section{Acknowledgements}

This work project was supported by National Science Foundation of China (No. 21346006 and 21473078), Department of scientific research project in Heilong jiang province (No.B201015), Scientific research project of Heilongjiang province education department (No.12541783 and 12541821), National college students' innovation and entrepreneurship training major project(No.201310222013), Key scientific research project of Jiamusi University(Sjz2012-19), Interdisciplinary research project of Jiamusi University (No.JC2014-005), Graduate scientific and technological innovation major project of Jiamusi University(No.LZR2014_034), Jiamusi University students' science and technology innovation project(No.XSYD 2004-020), Jiamusi University education scientific research project(JKY2014-042).

\section{References}

[1] Ye Rengao. Internal Medicine[M]. 6th ed, Beijing: People's medicine publishing house, 2004.

[2] Zou Meijuan. Studies on polysaccharide prodrugs of 5-aminosalicylic acid as potential colon-Specific delivery systems[D]. Shenyang: Shenyang Pharmaceutica University, 2004.

[3] Yang Zhixin, Du Sumei. Progress of 5-am inosalicylic acid preparation[J]. Anhui Medical and Pharmaceutical Journal, 2010, 14(2): 125-126.

[4] Kim H, Kim D, Choi D et al. Synthesis and properties of N,N-bis(5-am inosalicyl)-L-cystine as a colon-specific deliverer of 5-am inosalicylic acid and cystine[J]. DrugDeliv, 2008, 15(1): 37- 42.

[5] Jung Y.J., Lee J.S., Kim Y.M., Colon-specific prodrugs of 5-am inosalicylic acid:Systhesis and in vitro/in vivoproperties of acidic amino acid[J]. Pharm.Sci., 2001, 90(11): 1767-1775.

[6] Wang Guijie, Li Yan. Clinical effect of Mesalazine in the Treatment ulcerative colitis[J]. Practical Pharmacy And Clinical Remedies, 2006, 9(6): 361-365.

[7] Hu Fulian, Lin Gengjin, Yuan Arli, Xie Pengyan, Liu Jianxiang, Yuan Yaozong, Hu Yunbiao, Xu Guoming, Zhang Xiaojin, Yang Zhaoxu, Zhao Yingheng, Yang Jianquan, Zhou Lan. The efficacy and safety of mesalazine in the treatment of inflammatory bowel disease-A nationwide multicenter clinical trial[J]. Chinese Journal of New Drugs, 2001, 10(3): 201-203. 
[8] Munakata A, Yoshida Y, Muto T, et al. Double blind comparative study of sulfasalazine and controlled release mesalazine tablets in the treatment of active ulcerative colitis[J]. J Gastroenterol, 1995, 30(8): 108-111.

[9] Ad A van Bodegraven, Chris JJ Mulder. Indication for 5-aminosalicylate in inflammatory bowel disease:Is the body of evidence Complete[J]. Word J Gastroentenrol, 2006, 12(38): 6115-6123.

[10]Cottone M, Camma C. Mesalamine and relapse prevention on crohn's disease[J]. Gastronterology, 2000, 119: 597.

[11] Feagan B G.5-ASA therapy for active crohn's disease:old friends,old data,and a new conclusi on[J]. Clin Gastroenterol Hepatol, 2004, 2: 376-378.

[12] Wiersma H, Escher J C, Dilger K, Trenk D, Benninga M A, Van Boxtel C J, Taminiau J. Pharmaco kinetics of mesalazine pellets in children with inflammatory bowel disease[J]. Inflamm Bowel Dis, 2004, 10: 626-637.

[13] Brunner M, Greinwald R, Kletter K, Kvaternik H, Corrada ME, Eichler HG, Muller M. Gastrointestinal transit and release 5-aminosalicylic acid from 153Sm-labellede mesalazine Pell ets vs. tablets in male health volunteeers[J]. Aliment Pharmacol Ther, 2003, 17: 1163-1169.

[14] Deepak Parakkal, Eli D Ehrenpreis, Matthew P Thorpe, Karson S Butt, Bruce Hannon. A dynamic model of once-daily 5-aminosalicylic acid predicts clinical efficacy[J]. World $\mathrm{J}$ Gastroenterol, 2010, 16(1): 136-137.

[15] Wang Xigui, Wu Hongying, Weng Shifu, Wu Jinguang. Effects of Different Ligands on the Fluorescence Intensity of Tb(III)[J]. Spectroscopy and Spectral Analysis, 2007, 27(5): 991-994.

[16] Shishonok E.M., Leonchik S.V., Steeds J.W.et al. Strong ultraviolet luminescence from Cerium and gadolinium-doped cubic boron nitride[J]. Diam Relat Mater, 2007, 16(8): 1602-1607.

[17] Yumei Guo, Jinghe Yang, Xia Wu, Hongzhi Mao. Sensitive determination of protein based on the fluorescence enhancement effect of terbium(III)-epinephrine-protein-sodium dodecyl-sulfate system[J]. Luminesence, 2009, 24: 372-378. 